\title{
The tracking control of hypersonic aircraft attack angle based on the coupling of elastic form and engines
}

\author{
Junwei Lei ${ }^{1, a}$ and Yuliang Chen ${ }^{2}$ \\ ${ }^{1}$ Department of control engineering, Naval aeronautical and astronautical University \\ Yanti, 264001 \\ ${ }^{2}$ Department of Ordnance Science and Technology, Naval aeronautical and astronautical University \\ Yantai, China \\ aeijunwei@126.com
}

Keywords: hypersonic aircraft; stability; control; free flying; numerical simulation

\begin{abstract}
A kind of attack angle tracking controller with a former similar to PID control is proposed based on the pitch channel model of hypersonic aircraft released by America air force research center. Also the speed of the aircraft is controlled to tracking to a constant, which will make the system more stable and more easy to design a controller. At last, detailed numerical simulation is done to testify the rightness of the proposed method and the rightness of the hypersonic aircraft.
\end{abstract}

\section{Introduction}

The stable tracking control of attack angle is one of the most basic tasks in hypersonic control, its importance is that the stability of the attack angle can facilitate the control of engine and speed $^{[1-5]}$. It avoids the self-excited oscillation of engine thrust with attack angle . For traditional aircraft at low speed, it is relatively easy to control the stability of attack angle, the difficulty is the sensor measurement of attack angle and measurement of attack angle derivative. But stability control of the attack angle for hypersonic flight vehicle is not an easy thing. The tracking test of attack angle is proposed without considering the premise of sensor measurement difficulties, its significance lies in assuming that the attack angle can accurate measurement, the velocity of attack angle can be measured for the system to provide adequate damping.

The static instability of the hypersonic aircraft can be stabled by the traditional PID control strategy. If it can, then shows that the proposed model is reasonable and controllable; If not, it suggests that the model complexity and control difficulty have challenging, of course, it is also likely to show that model data has problem. Based on the above reasons, the attack stable tracing test flight simulation is carried out based on the United States air force announcing air-breathing hypersonic longitudinal motion model in this paper ${ }^{[6-10]}$. The results show that the static instability hypersonic aircraft can use PID control strategy to make the attack angle stable under the condition of the attack angle and derivative measurable.

\section{Model Description}

Considering the elastic shape structure, a kind of pitch channel hypersonic aircraft model built according to Lagrange equation is released by USA air force as followed:

$$
\begin{array}{r}
\dot{V}=\frac{T \cos \alpha-D}{m}-g \sin \gamma \\
\dot{\phi}=-2 \varsigma \omega_{n} \phi-\omega_{n}^{2} \phi+\omega_{n}^{2} \phi_{c} \\
\dot{\gamma}=\frac{L+T \sin \alpha}{m V}-\frac{g \cos \gamma}{V} \\
\dot{\alpha}=q-\dot{\gamma}
\end{array}
$$




$$
\begin{gathered}
\dot{q}=\frac{M}{I} \\
\dot{h}=V \sin \gamma
\end{gathered}
$$

And $V$ is speed, $\gamma$ is the speed angle, $\alpha$ is attack angle, $Q$ is the attitude angle speed, $h$ is the height. $\phi$ is the oil supplying factor, $\delta_{c}$ is the duck wing and $\delta_{e}$ is the lift rudder.

\section{Simulation settings of free flying}

In order to testify the rightness of the model of hypersonic aircraft, choose a initial height as $h_{0}=85000 * 0.3048$, initial speed as $V_{0}=7846 * 0.3048$, initial attack angle as $\alpha_{0}=0.0174$, and other initial state as: $\gamma_{0}=0, q_{0}=0, \eta_{10}=0.4588 * 0.3048 * 14.59, \eta_{20}=-0.08726 * 0.3048 * 14.59$, $\eta_{30}=-0.03671 * 0.3048 * 14.59$. .

Setting the throttle opening of engine for the switching control law of the PID control law with diffusion control law, and design

$$
\phi_{c}=k_{e t} e_{v}+k_{s e t} e_{v}+k_{d e} \dot{e}_{v}+k_{e t b} \frac{e_{v}}{\left|e_{v}\right|+\varepsilon_{e v}}, e_{v}>0, \phi_{c}=-300, e_{v}<0
$$

where $e_{v}=V^{d}-V, k_{e t}, k_{\text {set }}, k_{d e}, k_{e t b}$ and $\varepsilon_{e v}$ are the adjustable positive.

The expectations speed is $V^{d}=2391, \alpha^{d}=4 / 57.3$, error variables are $e_{\alpha}=\alpha-\alpha^{d}$, $u_{p}=k_{p} e_{\alpha}+k_{i} \int e_{\alpha} d t+k_{d} \dot{e}_{\alpha}$,

The rule of design canard and elevator are as follows:

$$
\delta_{c}=u_{p}-5, \delta_{e}==u_{p}-5
$$

Testing attack angle track dynamic characteristics of superb aircraft, setting $\dot{\eta}_{i}=0$,

The flight time is 10.5 seconds.

\section{Numerical Simulation and result analysis}

Set the ideal attack as $\alpha^{d}=4 / 57.3$ and the simulation results are as follows:

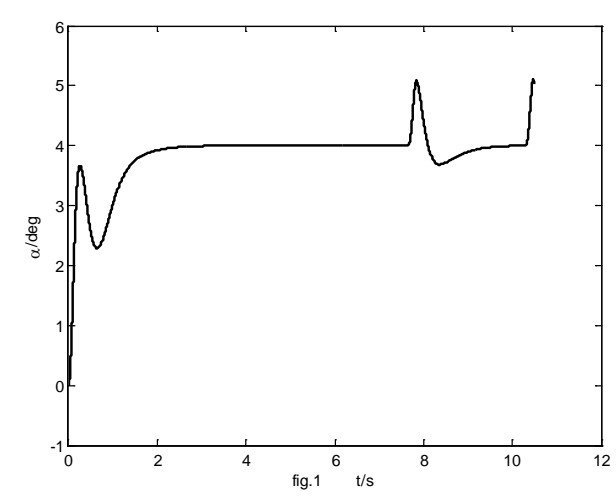

Fig 1 The curve of attack angle

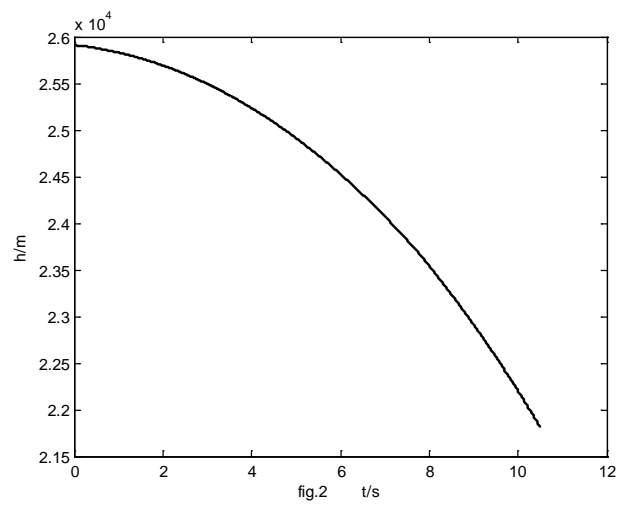

Fig 2 The curve of the height 


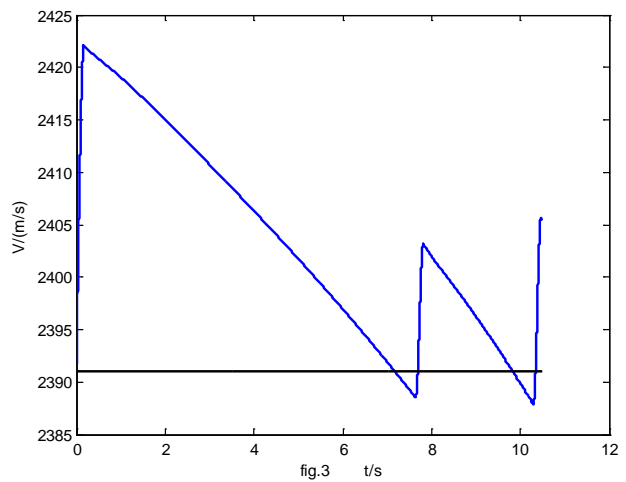

Fig 3 The curve of speed

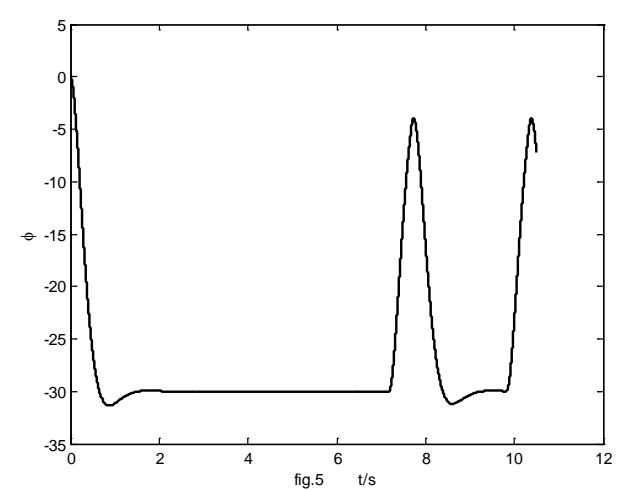

Fig 5 The curve of oil supplying factor

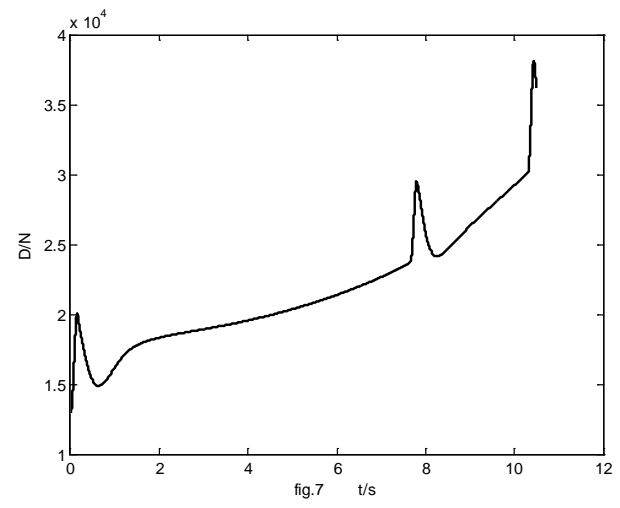

Fig 7 The curve of resistance

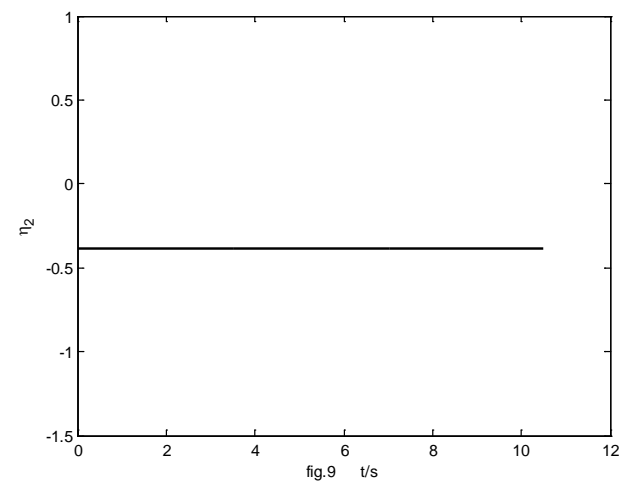

Fig 9 The state of second elastic shape

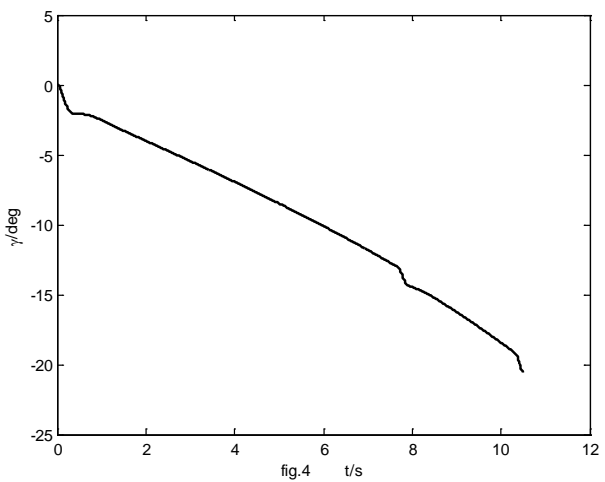

Fig 4 The curve of speed angle

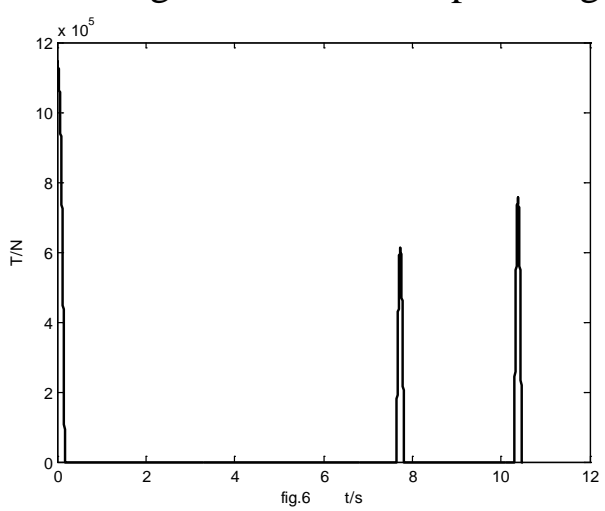

Fig 6 The curve of thrust

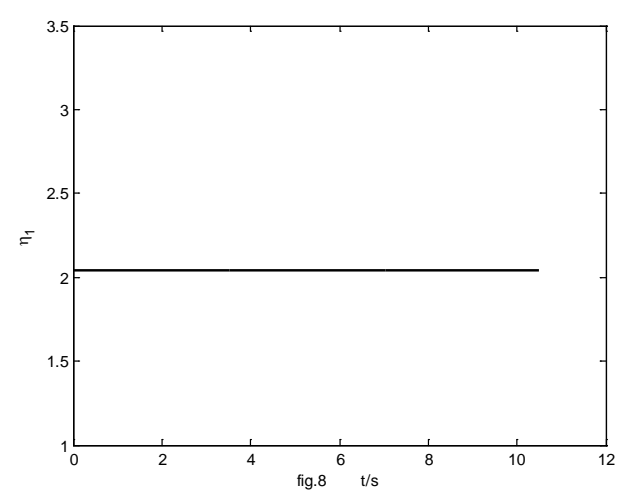

Fig 8 The state of first elastic shape

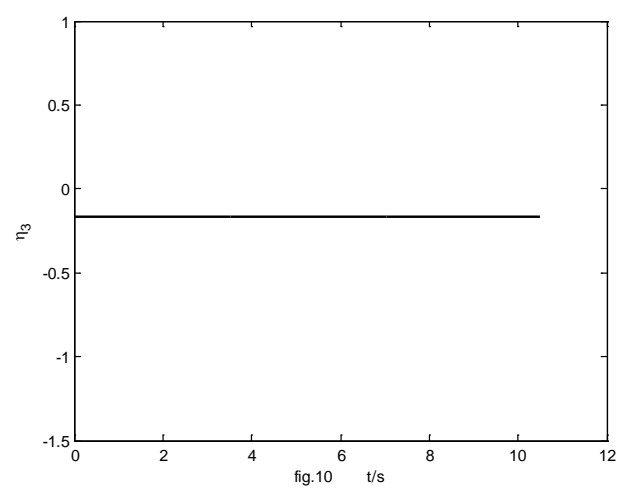

Fig 10 The state of third elastic shape 


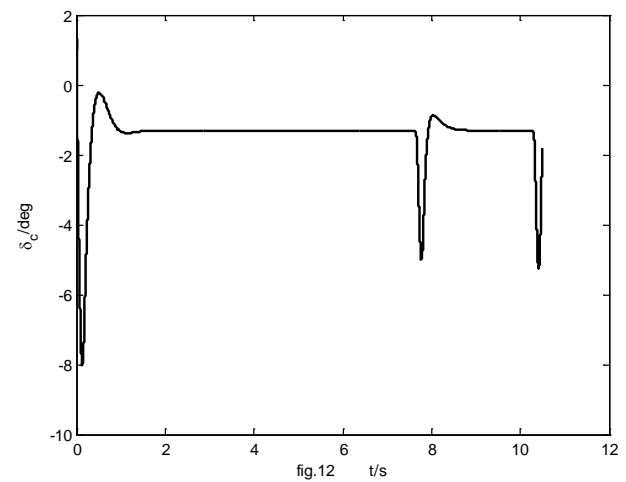

Fig 11 The curve of duck wing

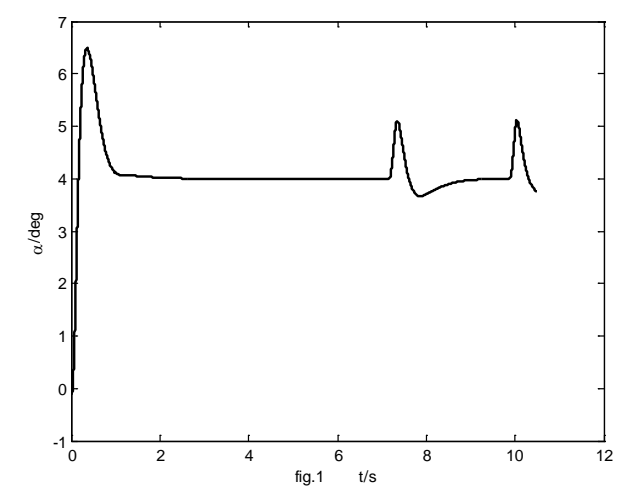

Fig 13 The curve of attack angle

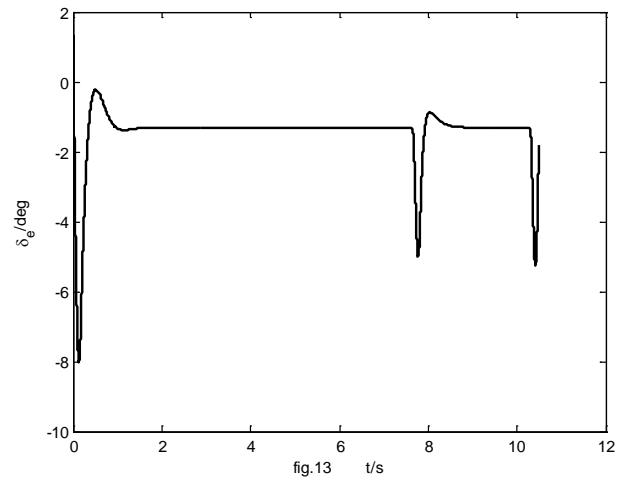

Fig 12 The curve of lift actuator

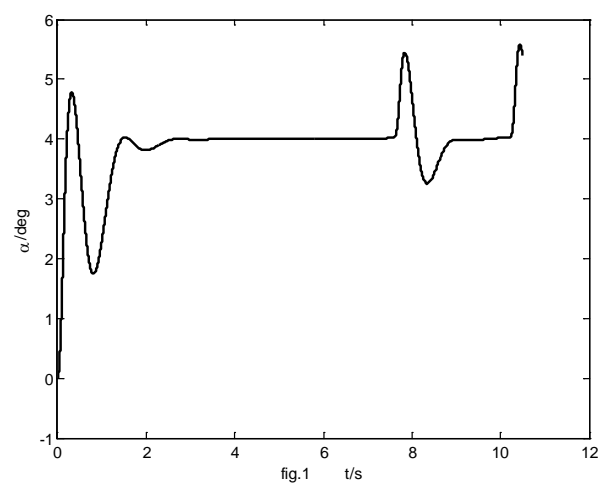

Fig 14 The curve of attack angle

When partial system is not be used, the curve of attack angle is as shown as figure 13. The overshoot amount of the first peak is larger, it can be seen that attack angle of hypersonic flight vehicle is asymmetric with negative attack angle. The constant amount of partial system is needed to reduce the overshoot, the stability time can also be increased at the same time. The attitude angular velocity is used to instead derivative of attack angle, then the simulation results are as figure 14. It indicates that the problem that derivative of attack angle is difficult to measure can be solved by using attitude angular velocity to approximate instead.

\section{Conclusion}

In this chapter, assuming that attack angle of hypersonic aircraft and its derivative are easy to measure, the PID control law with belt constant offset is used, the canard and elevator use synthetic compound control strategy, finally digital simulation program is used to verify the effectiveness of the designed PID control law by setting hypersonic flight vehicle constant speed with the elastic state based on the pitch channel model discussed in the previous section.It further verifies the rationality and controllability of the hypersonic model and data. Of course, follow-up studies will no longer use the derivative of attack angle in order to avoid the difficulty of its measurement. Finally, in order to try to the method that attitude angle rate insteads attack angle rate, and good results have been achieved, it shows that the damping of hypersonic aircraft attack angle control can be provided by the attitude angle velocity.

\section{References}

[1]Vaddi S S, Sengupta P. Controller design for hypersonic vehicles accommodating nonlinear state and control constraints, In: AIAA Guidance, Navigation, and Control Conference Chicago, USA, AIAA 2009-6286: 1-19.

[2]E. mooij. 1inear quadratic regulator reentry control-performance assessment using a taguchi approach. AIAA, international space planes and hypersonic systems and technologies 
conference. 1998: 665-677.

[3]Gregory I M, Mcminn J D and Shaughnessy J D. Hypersonic vehicle control law development using $\infty \mathrm{H}$ and $\mu$-synthesis [R]. NASA TM-4562, 1994.

[4]Lohsoonthorn P, Jonckheere E and Dalzell S. Eigenstructure vs constrained $\infty \mathrm{H}$ design for hypersonic winged cone [J]. Journal of Guidance, Control, and Dynamics, 2001, 24(4): 648-658.

[5]Appleby B D and Adams N J. Robust estimator design applied to the lateral dynamics of a hypersonic vehicle [A]. In: AIAA Guidance, Navigation, and Control Conference and Exhibit [C]. AIAA-1991-2689.

[6]Chavez F R and Schmidt D K.Uncertainy modeling for multivariable-control robustness analysis of elastic high-speed vehicles[J].Journal of Guidance, Control,and Dynamics,1999,22(1):87-95.

[7]Buschek $\mathrm{H}$ and Calise A J. Fixed order robust control design for hypersonic vehicles[A].In:AIAA Guidance, Navigation and Control Conference [C]. Washington,DC,AIAA 94-3662

[8] M. Ohno, Y. Yamaguchi, T. Hata, M. Takahama, Robust flight control law design for an automatic landing flight experiment [J], Control Engineering Practice, 1999, 7(9):1143-1152

[9]Naidu S D,Banda S S and Buffington J L.Unified approach to $\mathrm{H}_{2}$ and $\mathrm{H}_{\infty}$ optimal control of a hypersonic vehicle[A].In:Proceedings of the American control conference[C].San Diego, California,1999,vol.4:2737-2741.

[10]Wang Q, Stengel R F. Robust nonlinear control of a hypersonic aircraft [J]. Journal of Guidance, Control, and Dynamics, 2000, 23(4): 577-585. 